\title{
Non-local microscopic potentials for calculation of scattering observables of nucleons on deformed nuclei
}

\author{
Amine Nasri $^{1, \text { a }}$, Marc Dupuis ${ }^{1}$, Guillaume Blanchon ${ }^{1}$, Eric Bauge ${ }^{1}$, and Hugo F. Arellano ${ }^{2}$ \\ 1 CEA, DAM, DIF, 91297 Arpajon, France \\ 2 FCFM, University of Chile, Av. Blanco Encalada 2008, Santiago, Chile
}

\begin{abstract}
Direct reactions on deformed nuclei such as actinides are best studied with the coupled channel (CC) formalism and a complex coupling scheme. With all significant progress that has been made in describing target nuclei with mean field and beyond approaches, we can assess the scattering problem within CC framework using microscopic non-local potentials. To undertake this challenging task, one needs a welldefined strategy. In this work, we describe our choices of interaction, of microscopic description of target nuclei and our numerical methods to solve CC equations with non-local potentials. Motivations behind our choices are also presented.
\end{abstract}

\section{Introduction}

Recent developments in various areas like 4th generation reactors, and waste management have stressed the need for more accurate nuclear data on a wider energy range. A good understanding and prediction capacity of neutron scattering cross sections on minor actinides is crucial to all kinds of reactors based on fission processes. For deformed nuclei, the determination of direct reaction observables requires $\mathrm{CC}$ calculations. Local, phenomenological optical and transition potentials are the most commonly used in $\mathrm{CC}$ analyses, with the extrapolation issue associated when one uses such potentials in areas (energy range, target mass...) where there was no fitting procedure. Microscopic approches are being developped in order to improve prediction power and solve the aformentionned issue [1]. Potentials obtained microscopically are nonlocal, and recent studies [2-4] have emphasized the importance of treating explicitely this nonlocality, and not through a localization procedure. These developments lead to the need of a computation code capable of solving CC equations with non-local potentials. Along such a code, producing microscopic non-local optical and transition potentials and investigate effects of nonlocality in $\mathrm{CC}$ equations is also of interest. We briefly present in this document some successfull microscopic and phenomenological approaches to describe nucleon elastic and inelastic scattering on spherical nuclei, and our strategy to perform similar studies on deformed nuclei.

\section{Existing non-local potentials}

Optical and transition potentials that are derived from microscopic principles are non-local. Some phenomenological non-local potentials have been developed and successfully applied to the study of nucleon scattering of various target nuclei [5]. But in many studies, local or

a e-mail: amine.nasri@cea.fr localized potentials are used so as to reduce the numerical complexity when solving CC equations. However, it has been shown that this procedure has a significant impact on some scattering observables [6]. In an attempt to treat explicitely non-local effects while using microscopic calculations, Blanchon et al. [1] have derived an optical potential for the scattering of nucleons off ${ }^{40} \mathrm{Ca}$, using the Nuclear Structure Method (NSM) with the Gogny D1S interaction, and have successfully applied it to elastic scattering calculations for energies under $30 \mathrm{MeV}$.

Modern phenomenological non-local potentials have been developed to study charge asymmetry dependence of the optical potential [7] and transfer reactions [3]. All these studies show that it is possible and desirable to use nonlocal potentials when studying direct reactions. However, available codes like ECIS [8] use local potentials in CC equations.

\section{Inelastic scattering}

An example of calculation with microscopic non-local potentials to describe nucleon elastic and inelastic scattering is that of Dupuis et al. [9]. Starting from the Melbourne G matrix 2-body interaction [13], they have derived optical and transition potentials to study the scattering of nucleons off ${ }^{208} \mathrm{~Pb}$. They obtained ground state and transition 1-body density matrices using the Random-Phase Approximation (RPA) and performed their calculations with the DWBA98 code [10], which can use non-local potentials as input. This study did not use the $\mathrm{CC}$ framework, but the distorted wave Born approximation (DWBA) instead. Their calculations reproduce rather well experimental data for incident energies above $50 \mathrm{MeV}$ or so, given the fact that no fitting procedure was done througout the study.

Another successful semi microscopic approach is that of the JLM model, which has been applied to inelastic scattering of nucleons off ${ }^{238} \mathrm{U}$ for the lower excited states [11], using local potentials. In order to study deformed

(C) The Authors, published by EDP Sciences. This is an Open Access article distributed under the terms of the Creative Commons Attribution License 4.0 (http://creativecommons.org/licenses/by/4.0/). 

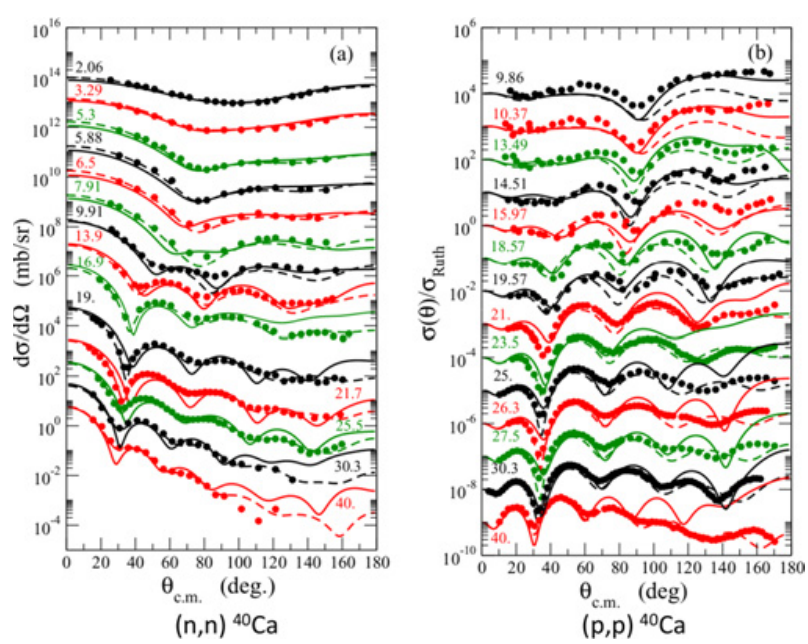

Figure 1. Elastic differential cross sections for (a) neutron and (b) proton scattering off ${ }^{40} \mathrm{Ca}$. Solid curve is from the NSM calculation, and dashed curve is Koning-Delaroche potential [1].

nuclei like ${ }^{238} \mathrm{U}$ with non-local potentials, one needs to write a computation code to solve $\mathrm{CC}$ equations without approximations like the DWBA.

\section{Strategy adopted}

The previously mentionned works, put together, bring forth the issue of solving $\mathrm{CC}$ equations with non-local potentials, and to develop all coupling potentials required to study nuclei of interest. To assess it, we have defined a strategy. Starting from a general formulation of the CC problem:

$$
\left\{\begin{array}{l}
\left(E+\frac{\hbar^{2}}{2 \mu} \vec{\nabla}^{2}-\left\langle\psi_{0}|\hat{U}| \psi_{0}\right\rangle\right)\left|u_{0}\right\rangle=\sum_{i \neq 0}\left\langle\psi_{0}|\hat{U}| \psi_{i}\right\rangle\left|u_{i}\right\rangle \\
\left(E^{\prime}+\frac{\hbar^{2}}{2 \mu} \vec{\nabla}^{2}-\left\langle\psi_{N}|\hat{U}| \psi_{N}\right\rangle\right)\left|u_{N}\right\rangle=\sum_{i \neq N}\left\langle\psi_{N}|\hat{U}| \psi_{i}\right\rangle\left|u_{i}\right\rangle
\end{array}\right.
$$

with $\mathrm{E}$ the incident energy, $\left|\psi_{0}\right\rangle$ the target nucleus' ground state, $\mu$ the reduced mass of the system, $|u\rangle$ the wave function describing the relative motion of the projectile and the target, $\hat{U}$ a potential energy operator, $E^{\prime}=E-E_{N}$, $E_{N}$ being the energy of the excited state $\left|\psi_{N}\right\rangle$, we describe our choices to solve this system. Required ingredients are the effective 2-body interaction and the microscopic description of the target nucleus.

\subsection{Numerical method to solve $\mathrm{CC}$ equations}

Following a method adopted to study charge exchange [12], we write a CC system for all possible values of the total angular momentum J, then we define Dyson integral equations by assuming for the propagator $\mathscr{G}$ the form (in radial coordinates):

$$
\mathscr{G}\left(r, r^{\prime}, k\right)=-\frac{i}{k} F\left(k r_{\text {inf }}\right)\left(F\left(k r_{\text {sup }}\right)-i G\left(k r_{\text {sup }}\right)\right)
$$

with $k$ the wave vector's norm, $r_{\text {inf }}=\min \left(r, r^{\prime}\right)$ and $r_{\text {sup }}=\max \left(r, r^{\prime}\right), \mathrm{F}$ and $\mathrm{G}$ the regular and irregular Coulomb wavefunctions. We solve numerically by matrix inversion methods to obtain phase shifts and then rebuild differential cross sections using Racah algebra. We have

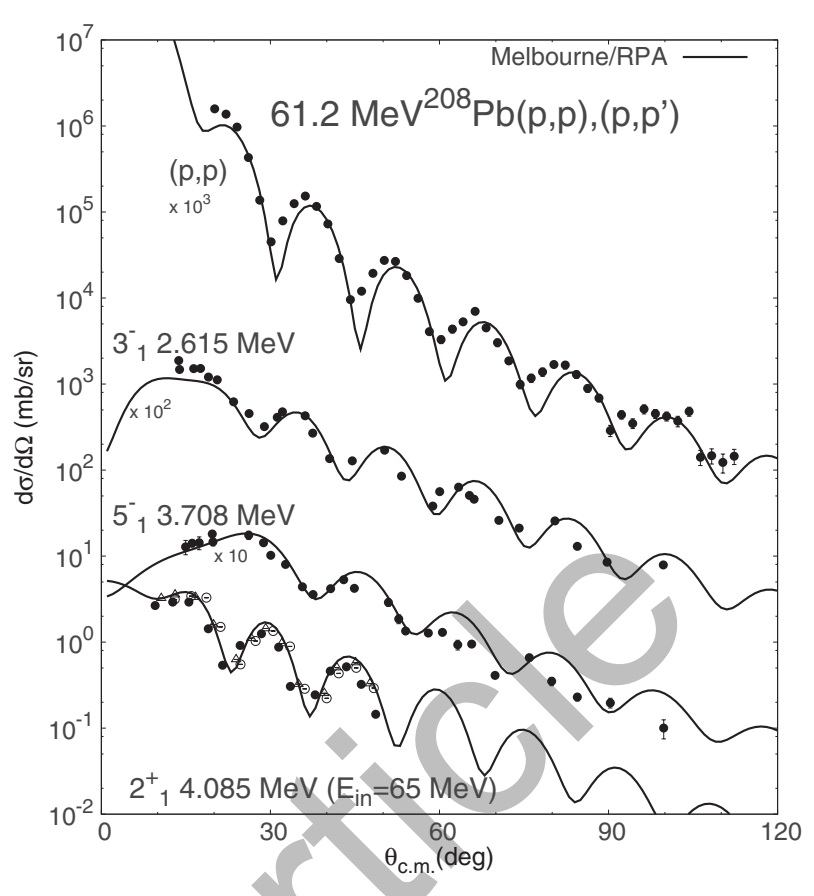

Figure 2. Differential cross sections for proton elastic and inelastic scattering off ${ }^{208} \mathrm{~Pb}$.

validated our code for elastic scattering and charge exchange, and the validation for inelastic scattering is in progress.

\subsection{Microscopic ingredients}

We have chosen to use the Melbourne G matrix as our 2-body effective interaction [13], given the good results in Refs. [9] and the possibility to extend the approach to other $\mathrm{G}$ matrices [14] in the future. In order to validate the computation code and our general approach, we have decided to start our study by focusing on ${ }^{208} \mathrm{~Pb}$, a double closed shell nucleus that is well described within the RPA. Therefore, our potentials for this nucleus use RPA ground state and transition 1-body density matrices. Once this validation procedure is completed, we will extend our study to ${ }^{238} \mathrm{U}$, an axially deformed nucleus which is now accurately described using a quasi particle random phase approximation (QRPA) approach [15], one that we shall follow.

\section{Perspectives}

The developments that are being performed and that will be performed throughout this project will allow us to investigate the impact of the non-locality in nucleonnucleus scattering within the CC framework. They will provide a modern tool for $\mathrm{CC}$ calculations using any type of potential, as for example potentials obtained using the NSM, with G matrices within a folding procedure, or phenomenological non-local potentials. Once operational, our new $\mathrm{CC}$ code will be able to use complex coupling schemes needed to predict direct inelastic scattering, especially for nucleon scattering off actinides at low incident energies. Moreover, it will become possible to account for couplings between 1-phonon and 2-phonon QRPA states using microscopic non-local potentials. This feature will enable a precise calculation of direct inelastic scattering to 2-phonon states which are needed 
to accurately take into account pre-equilibrium emissions above $10 \mathrm{MeV}$.

\section{References}

[1] G. Blanchon, M. Dupuis, H.F. Arellano, N. Vinh Mau, Phys. Rev. C 91, 014612 (2015)

[2] N. Keeley, R.S. Mackintosh, Phys. Rev. C 90, 044602 (2014)

[3] L.J. Titus, F.M. Nunes, and G. Potel, Phys. Rev. C 93, 014604 (2016)

[4] A. Ross, L.J. Titus, F.M. Nunes, M.H. Mahzoon, W.H. Dickhoff, R.J. Charity, Phys. Rev. C 92, 044607 (2015)

[5] F. Perey, B. Buck, Nucl. Phys. 32, 353 (1962)

[6] N. Austern, Phys. Rev. 137, B752 (1965)

[7] M.H. Mahzoon, Ph.D. Thesis (Washington University, St Louis, 2015)
[8] J. Raynal, Notes on ECIS94 (CEA Saclay Report No. CEA-N-2772, Gif-sur-Yvette, 1994)

[9] M. Dupuis, S. Karataglidis, E. Bauge, J.P. Delaroche, D. Gogny, Phys. Lett. B 665, 152 (2008)

[10] Jacques Raynal, Computer code DWBA98 (NEA 1209/05, 1998)

[11] E. Bauge, J.P. Delaroche, M. Girod, Nucl. Phys. A 654, 829 (1999)

[12] H.F. Arellano, W.G. Love, Phys. Rev. C 76, 014616 (2007)

[13] K. Amos, P.J. Dortmans, H.V. von Geramb, S. Karataglidis et J. Raynal, Adv. Nucl. Phys. 25, 275 (2000)

[14] H.F. Arellano, J.-P. Delaroche, Eur. Phys. J. A 51 (2015)

[15] S. Péru, G. Gosselin, M. Martini, M. Dupuis, S. Hilaire, J.-C. Devaux, Phys. Rev. C 83, 014314 (2011) 